\title{
Motor-Imagery EEG Signals Classificationusing SVM, MLP and LDA Classifiers
}

\author{
Yogendra Narayan ${ }^{1}$ \\ ${ }^{1}$ Department of ECE, Chandigarh University, Gharuan, Mohali, Punjab-140413, India \\ narayan.yogendra1986@gmail.com
}

\begin{abstract}
Article History: Received: 11 January 2021; Accepted: 27 February 2021; Published online: 5 April 2021
Abstract:Electroencephalogram (EEG)signals based brain-computer interfacing (BCI) is the current technology trends in the field of rehabilitation robotic. This study compared the performance of support vector machine (SVM), linear discriminant analysis (LDA) and multi-layer perceptron (MLP) classifier with the combination of eight different features as a feature vector. EEG data were acquired from 20 healthy human subjects with predefined protocols. After the EEG signals acquisition, it was pre-processed followed by feature extraction and classification by using SVM MLP and LDA classifiers. The results exhibited that the SVM method was the best approach with $98.8 \%$ classification accuracy followed by MLP classifier. Finally, the SVM classifier and Arduino Mega controller was employed for offline controlling of the gripper of the robotic arm prototype. The finding of this study may be useful for online controlling as well as multi-degree of freedom with multi-class EEG dataset.

Keywords: Motor-Imagery, EEG signal, SVM, MLP, LDA, PCA.
\end{abstract}

\section{Introduction}

Neural activities of the brain are shown in the form of EEG signals which are captured with the help of multiple-electrode EEG machines either over the cortex under the skull (inside the brain) or over the scalp[1]. The representation of EEG signal is done in the time domain whereas, few EEG measuring devices are available which can perform some signal processing steps to obtain frequency analysis along with imaging tools to visualize EEG topographies [2], [3].

Generally, EEG signals are considered as the projection of neural activities that usually gets attenuated by dura mater, leptomeninges, scalp, cerebrospinal fluid, and the galea[4]. It is difficult to understand and locate the rhythms of the brain [5]. So, the advanced technology and processing tools[6] must have the ability to isolate the desired waveforms from the EEG signals and then analyze it[7].EEG system comprises delicate electrodes, filters, needle-type registers and set of the differential amplifier[8]. The EEG signals can be graphically represented on the paper. It was observed that the signal must be in digital form for analysis and it requires the sampling, quantization, and encoding of the signal [9], [10]. The computerized system permits simulations, variable settings, sampling frequency and some advanced processing equipment [11], [12], [6].

So, the EEG signals are converted from analogue into the digital form by using analogue-to-digital converters (ADC)[13], [14]. EEG signal has a bandwidth of approximately $100 \mathrm{~Hz}$. Hence, the minimum sampling frequency of 200 samples/sec is required for sampling the EEG signals[15].EEG signals undergo the process of quantization to preserve diagnostic information[16]. Each signal sample is represented by up to 16 bits for accurate recording[17]. This provides the required memory volume for epileptic seizure monitoring records and storing the signals massively[18]. Generally, the memory size for storing the EEG signal is much smaller than that used for storing the radiological images [19][20], [21].

This work computes the performance of the total eight features to classify the EEG signals for discriminating left hand and right-hand movements by using SVM, MLP and LDAclassifiers. The whole work is divided into four parts, the first one is the introduction part, and Second part presents the materials and methods while the third part describes the results and discussion. Finally, the conclusion of work is presented in section 4.

\section{MATERIALS AND METHODS}

\subsection{EEG data acquisition}

EEG data were acquired from 20 healthy subjects at Bio-Medical Laboratory of NITTTR Chandigarh, India[22], [23]. After the raw EEG signal acquisition, EEG data was passed through a $4^{\text {th }}$ order band-pass Butterworth filter $(8 \mathrm{~Hz}$ to the $30 \mathrm{~Hz}$ range) for noise elimination[24]. Further, a notch filter of cut off frequency $50 \mathrm{~Hz}$ was employed for power line interference. Ocular artifacts were rejected by spatial filtering based on ICA algorithm[25]. After these steps, suitable features are extracted with the help of the CSP method followed by dimension reduction by PCA[26]. Finally, the classification is done and accuracy is used for performance comparison [27]. Fig. 1 shows the complete experimental setup for EEG data recording from a healthy human subject. 


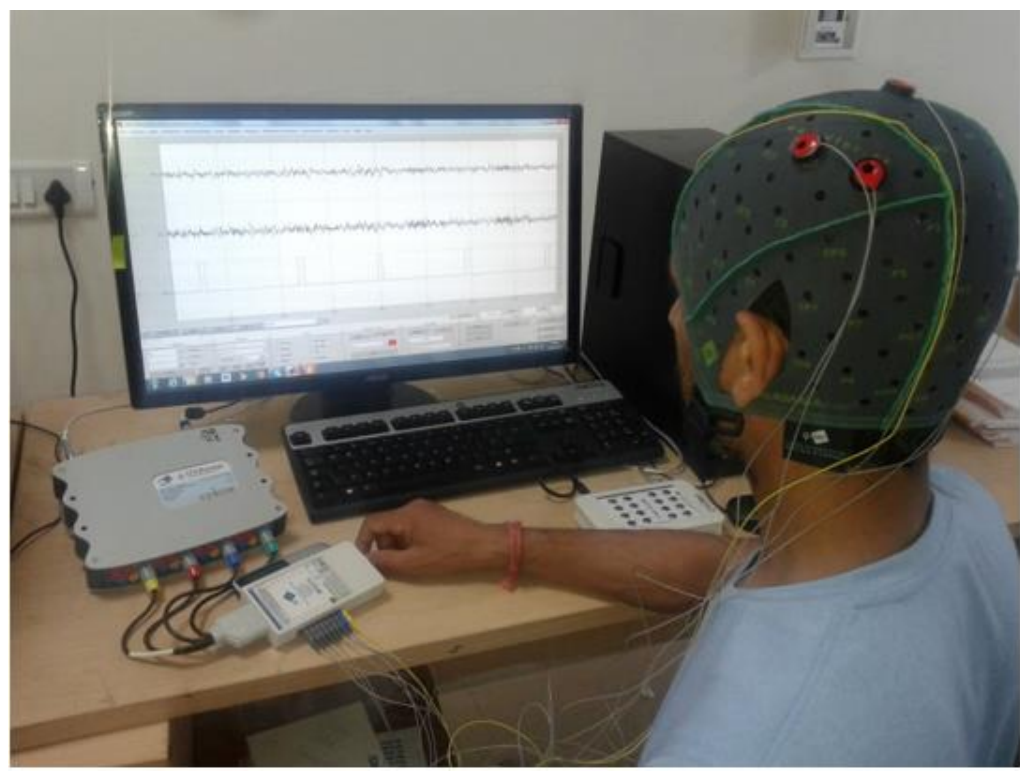

Fig. 1 EEG data recording for left and right-hand movements

\subsection{EEG feature extraction}

Total eight features were extracted namely as AAR parameter,Barlow parameter, Hjorth parameter, Temporal and Spatial Complexity (TSC), Running Fractal Dimension (RFD), minimum energy, band power, andvariance. Variance is calculated with the window length 500 millisecond (ms) with $492 \mathrm{~ms}$ overlapping and the band power is calculated for alpha and beta region whereas signal to noise ratio is computed by using minimum energy approach. EEG data sequence complexity is measured by TSC.Higuchi's algorithm is used for RFD calculation [28], Hjorth parameter, Barlow parameter, AAR parameter are calculated[29].Fig. 2 shows the four channels EEG signals recorded in temporal form from a healthy human subject. Table 1 shows the features utilized for EEG signal classification for discriminating the left and right-hand movements to control the robotic arm prototype.

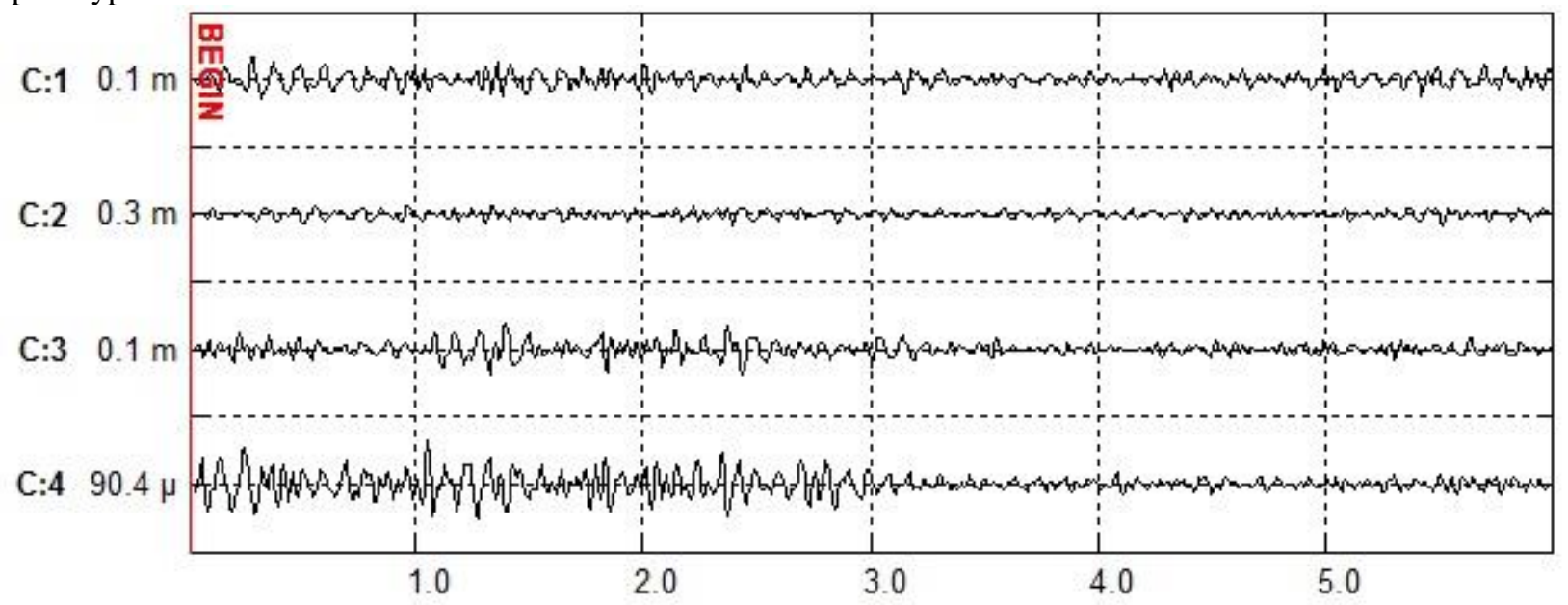

Fig. 2. Four-Channel EEG data recorded from the healthy human subject in temporal form Table 1. Mathematical Definitions of Features

\begin{tabular}{|r|l|c|}
\hline \multicolumn{1}{l}{$\begin{array}{l}\text { S. } \\
\text { No. }\end{array}$} & features & definition \\
\hline 1 & Activity $\left(\mathrm{A}^{2}\right)$ & $\mathrm{A}^{2}=\frac{1}{\mathrm{~T}} \int_{\mathrm{t}-\mathrm{T}}^{\mathrm{t}} \mathrm{x}(\mathrm{t})^{2} \mathrm{dt}$ \\
\hline 2 & Mobility & $D^{2}=\frac{1}{\mathrm{~T}} \int_{\mathrm{t}-\mathrm{T}}^{\mathrm{t}}\left(\frac{\mathrm{dx}(\mathrm{t})}{\mathrm{dt}}\right)^{2} \mathrm{dt}$ \\
& & Mobility $=\sqrt{\frac{D^{2}}{\text { Activity }}}$ \\
\hline
\end{tabular}




\begin{tabular}{|r|c|c|}
\hline 3 & Complexity & Complexity $=\sqrt{\frac{\frac{1}{\mathrm{~T}} \int_{\mathrm{t}-\mathrm{T}}^{\mathrm{t}}\left(\frac{\mathrm{d}^{2} \mathrm{x}(\mathrm{t})}{\mathrm{dt}^{2}}\right)^{2} \mathrm{dt}}{\frac{1}{\mathrm{~T}} \int_{\mathrm{t}-\mathrm{T}}^{\mathrm{t}}\left(\frac{\mathrm{dx}(\mathrm{t})}{\mathrm{dt}}\right)^{2} \mathrm{dt}}}$ \\
\hline 4 & $\begin{array}{l}\text { Mean Amplitude } \\
(\mathrm{MA})\end{array}$ & $M A=\frac{1}{\mathrm{~T}} \int_{\mathrm{t}-\mathrm{T}}^{\mathrm{t}}|\mathrm{x}(\mathrm{t})| \mathrm{dt}$ \\
\hline 5 & $\begin{array}{c}\text { Mean Frequency } \\
(\mathrm{MF})\end{array}$ & $M F=\frac{E\left|\frac{d x(t)}{d t}\right|}{E|x(t)|}$ \\
\hline 6 & $\begin{array}{l}\text { Spectral Purity Index } \\
\text { SPI }\end{array}$ & SPI $=\frac{E\left|\frac{d x(t)}{d t}\right|^{2}}{E\left|\frac{d^{2} x(t)}{d t^{2}}\right| E|x(t)|}$ \\
\hline 8 & $\begin{array}{c}6^{\text {th }} \text { orders } \\
\text { parameter }\end{array}$ & AAR \\
\hline variance & $x_{k}-a_{1, k} x_{k-1}-\cdots a_{p, k} x_{k-p}=\varepsilon_{k}$ \\
\hline
\end{tabular}

\subsection{Classifiers}

After extracting the features, a feature vector was formed to classify the EEG data whose dimension was reduced by PCA approach. In this study, SVM, MLP and LDAclassifier was compared to each other[30], [31], [32]. Threefold cross-validation technique was applied for achieving the classification accuracy[33]. In threefold cross-validation technique, the whole dataset was divided into three equal parts in which two parts were used for training the classifier whereas one part of data was utilized for testing purpose and no part of data was used for validation the classifiers[34], [35]. Fig. 3 shows the block diagram representation of the complete workflow.

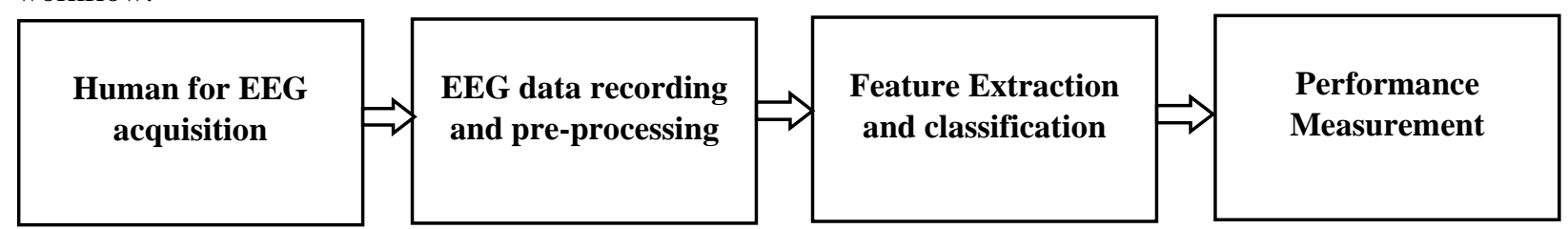

Fig 3. Complete block diagram of EEG signal classification model

\section{RESULTS}

In this study, the performance of the total of three classifiers namely SVM, MLP and LDA were compared with a total of eight features. Threefold cross-validation method was employed for computing the classification accuracy of all classifiers. Accuracy for 768 number of sample was taken to decide the final results for comparison purpose. Finally, the best classifier was chosen for controlling the two movements of the robotic arm (gripper open and close operations).Fig 4 shows the complete structure of MI-based movement controlling of robotic arm prototype.

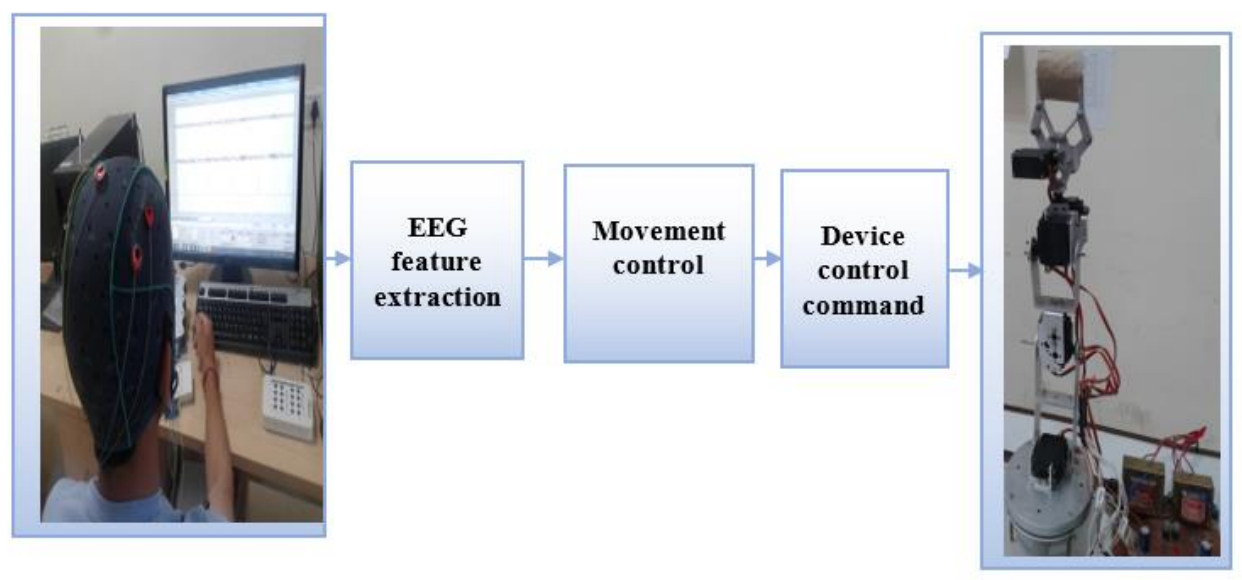

Fig. 4 Controlling of Assistive Devices Using MI-based EEG Signals 
The classification accuracy of the LDA method was presented in Table 2 in which the error for class 1 and class 2 was $0 \%$ and $9.4 \%$ thereby indicating the good discrimination capability of approach. Initially when the number of samples for classification was 128 then the total error was $50 \%$ thereby indication $50 \%$ classification accuracy. Finally, it achieved a $91.6 \%$ overall accuracy.

Table 2. Performanceof LDA classifier for EEG signals classification.

\begin{tabular}{|c|c|c|c|c|}
\hline $\begin{array}{c}\text { No of } \\
\text { Sample }\end{array}$ & $\begin{array}{c}\text { Error } \\
\text { class1 }(\%)\end{array}$ & $\begin{array}{c}\text { Error } \\
\text { class 2 }(\%)\end{array}$ & $\begin{array}{c}\text { Total } \\
\text { error }(\%)\end{array}$ & $\begin{array}{c}\text { Overall } \\
\text { Accuracy } \\
(\%)\end{array}$ \\
\hline 128 & 0.0 & 50.0 & 50 & 50.0 \\
\hline 256 & 22.5 & 21.9 & 44.4 & 55.6 \\
\hline 384 & 20.0 & 16.9 & 36.9 & 63.1 \\
\hline 512 & 21.3 & 20.6 & 41.9 & 59.1 \\
\hline 640 & 13.8 & 14.4 & 28.1 & 71.9 \\
\hline 768 & 0.0 & 9.4 & 9.4 & 91.6 \\
\hline
\end{tabular}

The classification results of the SVM classifier was presented in Table 3. The table shows the $98.8 \%$ classification accuracy. When EEG data samples were 128, accuracy was 50\%. But when the number of samples increased gradually then it achieved the higher classification accuracy and at last $98.8 \%$ accuracy was achieved by SVM classifier. The performance of SVM classifier was found best as compared to other classifiers used in this work.

Table 3.Performance of SVM classifier for EEG signals classification.

\begin{tabular}{|c|c|c|c|c|}
\hline $\begin{array}{c}\text { No of } \\
\text { Sample }\end{array}$ & $\begin{array}{c}\text { Error } \\
\text { class1 }(\%)\end{array}$ & $\begin{array}{c}\text { Error } \\
\text { class 2 }(\%)\end{array}$ & $\begin{array}{r}\text { Total } \\
\text { error }(\%)\end{array}$ & $\begin{array}{c}\text { Overall } \\
\text { Accuracy } \\
(\%)\end{array}$ \\
\hline 128 & 0.0 & 50.0 & 50.0 & 50.0 \\
\hline 256 & 1.5 & 46.9 & 48.4 & 51.6 \\
\hline 384 & 0.0 & 50.0 & 50.0 & 50.0 \\
\hline 512 & 0.0 & 50.0 & 50.0 & 50.0 \\
\hline 640 & 0.0 & 28.1 & 28.1 & 71.9 \\
\hline 768 & 0.0 & 1.2 & 1.2 & 98.8 \\
\hline
\end{tabular}

The classification performance of the MLP method was shown in Table 4. If the number of EEG data samples were 128 then accuracy was 50\%. With 640 number of samples, accuracy increased up to 75\% and finally, with 768 number of samples MLP classifier achieved 95\% classification accuracy. MLP classifier was found second-best classifier with the eight feature combination in the form of feature vector whereas LDA was found the least performer. It is clear from the above discussion that SVM classifier was best with the given feature vector and utilized for controlling the robotic arm prototype.

Table 4. Performanceof MLP classifier for EEG signals classification.

\begin{tabular}{|c|c|c|}
\hline No of Sample & $\begin{array}{c}\text { Total error } \\
(\%)\end{array}$ & $\begin{array}{c}\text { Overall } \\
\text { Accuracy }(\%)\end{array}$ \\
\hline 128 & 50.0 & 50.0 \\
\hline 256 & 56.9 & 43.1 \\
\hline 384 & 44.4 & 55.6 \\
\hline 512 & 41.3 & 59.7 \\
\hline 640 & 25.0 & 75.0 \\
\hline 768 & 5.0 & 95.0 \\
\hline
\end{tabular}

\section{CONCLUSION}

This work compared the performance of three classifiers namely SVM MLP and LDA with eight different features combination in the form of the feature vector. In this context, 20 healthy human EEG dataset was acquired and pre-processed before the features extraction process. Finally, all feature were combined in the form 
of a feature vector and applied to all classifiers for comparison purpose. The results showed that SVM classifier was found best among all classifier with the given feature vector. Therefore SVM classifier was utilized for actuating the robotic arm prototype. Results also showed the successful controlling for gripper open and close operation with SVM classifier and Arduino Mega controller.In near future, the multimodal data-based technique can be used for simultaneous recognition of hand, leg and finger movement with modular control facilities in which EEG signals will be fused with EMG and EOG signals depending upon the user's requirement for developing the assistive technology.

\section{References}

1. M. Wang, J. Hu, and H. A. Abbass, "BrainPrint: EEG Biometric Identification based on Analyzing Brain Connectivity Graphs,” Pattern Recognit., vol. 300, no. 5, p. 107381, 2020.

2. D. Bhati, M. Sharma, R. B. Pachori, and V. M. Gadre, "Time-frequency localized three-band biorthogonal wavelet filter bank using semidefinite relaxation and nonlinear least squares with epileptic seizure EEG signal classification," Digit. Signal Process. A Rev. J., vol. 62, pp. 259-273, 2017.

3. O. W. Samuel, Y. Geng, X. Li, and G. Li, "Towards Efficient Decoding of Multiple Classes of Motor Imagery Limb Movements Based on EEG Spectral and Time Domain Descriptors," J. Med. Syst., vol. 41, no. 12, 2017.

4. E. Monge-Pereira, J. Ibañez-Pereda, I. M. Alguacil-Diego, J. I. Serrano, M. P. Spottorno-Rubio, and F. Molina-Rueda, "Use of Electroencephalography Brain-Computer Interface Systems as a Rehabilitative Approach for Upper Limb Function After a Stroke: A Systematic Review,” $P M \& R$, vol. 9, no. 9, pp. 918932, 2017.

5. H. Ji, J. Li, R. Lu, R. Gu, L. Cao, and X. Gong, "EEG Classification for Hybrid Brain-Computer Interface Using a Tensor Based Multiclass Multimodal Analysis Scheme," Comput. Intell. Neurosci., vol. 2016, 2016.

6. M. Kaur and V. Wasson, "ROI Based Medical Image Compression for Telemedicine Application," in Procedia Computer Science, 2015, vol. 70, pp. 579-585.

7. J. Minguillon, M. A. Lopez-Gordo, and F. Pelayo, "Trends in EEG-BCI for daily-life: Requirements for artifact removal," Biomed. Signal Process. Control, vol. 31, pp. 407-418, 2017.

8. N. Hooda, R. Das, and N. Kumar, "Fusion of EEG and EMG signals for classification of unilateral foot movements," Biomed. Signal Process. Control, vol. 60, p. 101990, 2020.

9. Y. Narayan, V. Ahlawat, and S. Kumar, "Pattern recognition of sEMG signals using DWT based feature and SVM Classifier,” Int. J. Adv. Sci. Technol., vol. 29, no. 10, pp. 2243-2256, 2020.

10. Y. Narayan, D. Kumar, and S. Kumar, "Comparative analysis of sEMG signal classification using different K-NN algorithms,” Int. J. Adv. Sci. Technol., vol. 29, no. 10, pp. 2257-2266, 2020.

11. L. Minati, N. Yoshimura, and Y. Koike, "Hybrid Control of a Vision-Guided Robot Arm by EOG, EMG, EEG Biosignals and Head Movement Acquired via a Consumer-Grade Wearable Device," IEEE Access, vol. 4, no. 8, pp. 9528-9541, 2016.

12. Y. Narayan, R. M. Singh, L. Mathew, and S. Chatterji, "Surface EMG Signal Classification Using Ensemble Algorithm, PCA and DWT for Robot Control," in International Conference on Advanced Informatics for Computing Research. Springer, Singapore, 2019, vol. 10, pp. 424-434.

13. L. W. Ko, S. S. K. Ranga, O. Komarov, and C. C. Chen, "Development of Single-Channel Hybrid BCI System Using Motor Imagery and SSVEP," J. Healthc. Eng., vol. 2017, 2017.

14. Y. Narayan, L. Mathew, and S. Chatterji, "SEMG signal classification with novel feature extraction using different machine learning approaches," J. Intell. Fuzzy Syst., vol. 35, no. 5, pp. 5099-5109, 2018.

15. B. Kim, L. Kim, Y. H. Kim, and S. K. Yoo, "Cross-association analysis of EEG and EMG signals according to movement intention state,” Cogn. Syst. Res., vol. 44, pp. 1-9, 2017.

16. R. Zarei, J. He, S. Siuly, and Y. Zhang, "A PCA aided cross-covariance scheme for discriminative feature extraction from EEG signals," Comput. Methods Programs Biomed., vol. 146, pp. 47-57, 2017.

17. A. Al-Ani, I. Koprinska, and G. Naik, "Dynamically identifying relevant EEG channels by utilizing channels classification behaviour," Expert Syst. Appl., vol. 83, pp. 273-282, 2017.

18. A. Arunkumar et al., "Classification of focal and non focal EEG using entropies," Pattern Recognit. Lett., vol. 94, pp. 112-117, 2017.

19. D. R. Toledo, G. M. Manzano, J. A. Barela, and A. F. Kohn, "Cortical correlates of response time slowing in older adults: ERP and ERD/ERS analyses during passive ankle movement," Clin. Neurophysiol., vol. 127, no. 1, pp. 655-663, 2016.

20. B. Goyal, A. Dogra, S. Agrawal, B. S. Sohi, and A. Sharma, "Image denoising review: From classical to state-of-the-art approaches," Inf. FUSION, vol. 55, pp. 220-244, Mar. 2020.

21. N. Mittal, U. Singh, and B. S. Sohi, "A novel energy efficient stable clustering approach for wireless sensor networks," Wirel. Pers. Commun., vol. 95, no. 3, pp. 2947-2971, 2017. 
22. Babita, P. Kumari, Y. Narayan, and L. Mathew, "Binary movement classification of sEMG signal using linear SVM and Wavelet Packet Transform," in 1st IEEE International Conference on Power Electronics, Intelligent Control and Energy Systems, ICPEICES 2016, 2017.

23. Y. Narayan, L. Mathew, and S. Chatterji, "sEMG signal classification using Discrete Wavelet Transform and Decision Tree classifier," Int. J. Control Theory Appl., vol. 10, no. 6, pp. 511-517, 2017.

24. P. Virdi, Y. Narayan, P. Kumari, and L. Mathew, "Discrete Wavelet Packet based Elbow Movement classification using Fine Gaussian SVM," in 1st IEEE International Conference on Power Electronics, Intelligent Control and Energy Systems, ICPEICES 2016, 2017, pp. 1-5.

25. O. W. Samuel, X. Li, Y. Geng, P. Feng, and S. Chen, "Motor Imagery Classification of Upper Limb Movements Based on Spectral Domain Features of EEG Patterns," Eng. Med. Biol. Soc. (EMBC), 39th Annu. Int. Conf. IEEE, pp. 2976-2979, 2017.

26. S. Aliakbaryhosseinabadi, E. N. Kamavuako, N. Jiang, D. Farina, and N. Mrachacz-Kersting, "Classification of EEG signals to identify variations in attention during motor task execution," J. Neurosci. Methods, vol. 284, pp. 27-34, 2017.

27. C. Vidaurre, C. Klauer, T. Schauer, A. Ramos-Murguialday, and K. R. Müller, "EEG-based BCI for the linear control of an upper-limb neuroprosthesis," Med. Eng. Phys., vol. 38, no. 11, pp. 1195-1204, 2016.

28. J. Virkkala and S. Himanen, "Fractal dimension of EEG in sleep onset," Proc. 3rd Eur. ..., no. Table 1, pp. $5-8,2002$.

29. A. K. Mukhopadhyay and S. Samui, "An experimental study on upper limb position invariant EMG signal classification based on deep neural network," Biomed. Signal Process. Control, vol. 55, no. 5, pp. 1-8, 2020.

30. X. Yu, P. Chum, and K. B. Sim, "Analysis the effect of PCA for feature reduction in non-stationary EEG based motor imagery of BCI system," Optik (Stuttg)., vol. 125, no. 3, pp. 1498-1502, 2014.

31. J. U. Chu, I. Moon, and M. S. Mun, "A real-time EMG pattern recognition system based on linearnonlinear feature projection for a multifunction myoelectric hand," IEEE Trans. Biomed. Eng., vol. 53, no. 11, pp. 2232-2239, 2006.

32. A. Phinyomark, H. Hu, P. Phukpattaranont, and C. Limsakul, "Application of linear discriminant analysis in dimensionality reduction for hand motion classification," Meas. Sci. Rev., vol. 12, no. 3, pp. 82-89, 2012.

33. Babita, P. Kumari, Y. Narayan, and L. Mathew, "Binary movement classification of sEMG signal using linear SVM and Wavelet Packet Transform," in 1st IEEE International Conference on Power Electronics, Intelligent Control and Energy Systems, ICPEICES 2016, 2016, pp. 2-5.

34. P. Kumari, Y. Narayan, V. Ahlawat, and L. Mathew, "Advance approach towards elbow movement classification using discrete wavelet transform and quadratic support vector machine," in Communication and Computing Systems (C) 2017 Taylor \& Francis Group, London, ISBN 978-1-138-02952-1 Advance, 2017, pp. 839-844.

35. C. Garg, Y. Narayan, and L. Mathew, "Development of a software module for feature extraction and classification of EMG signals," in 2015 Communication, Control and Intelligent Systems (CCIS), 2015 , vol. 1, pp. 250-254. 